\title{
Tumores cutáneos de colisión
}

\section{Cutaneous collision tumors}

Carla Barbini ${ }^{1}$, Matías Stringa ${ }^{2}$, Aníbal María Parigini ${ }^{3}$, Javier Anaya $^{4}$, Raúl Valdez $^{5}$ y Corina Isabel Busso ${ }^{6}$

\begin{tabular}{|c|c|}
\hline & \\
\hline $\begin{array}{l}\text { Los tumores de colisión consisten en neoplasias compuestas por dos } \\
\text { poblaciones celulares distintas que mantienen una clara diferencia- } \\
\text { ción de sus bordes y que se encuentran adyacentes una de otra en } \\
\text { la misma muestra histopatológica. Esta asociación puede corres- } \\
\text { ponder a dos tumores malignos, dos benignos o uno maligno y uno } \\
\text { benigno. Son infrecuentes y, en ocasiones, representan un desafío } \\
\text { clínico para la detección correcta de ambas neoplasias. Se presen- }\end{array}$ & $\begin{array}{l}\text { tan los casos de tres pacientes con tumores cutáneos de colisión, de } \\
\text { estirpe melanocítica combinada con queratinocítica; en dos de ellos } \\
\text { ambas neoplasias fueron malignas y en uno, se asociaron una lesión } \\
\text { maligna y una benigna. } \\
\text { Palabras clave: tumores de colisión, neoplasias de colisión. } \\
\text { Dermatol. Argent. } 2021,27 \text { (2): 59-63 }\end{array}$ \\
\hline
\end{tabular}

\section{ABSTRACT}

Collision tumors consist of neoplasms composed of two different cell populations that maintain a clear differentiation of their borders, and that are adjacent to each other in the same histopathological sample. This association can correspond to two malignant tumors, two benign, or one malignant and one benign. They are infrequent and, at times, represent a clinical challenge for the correct detection of both neoplasms.
Three cases of cutaneous collision tumors of a melanocytic line combined with a keratinocytic line are presented, two of them in which both neoplasms were malignant and one that associated a malignant and a benign lesion. Key words: collision tumors, collision neoplasm.

Dermatol. Argent. 2021, 27 (2): 59-63

\footnotetext{
1 Médica Residente, Servicio de Dermatología

2 Médico de Planta, Servicio de Dermatología

3 Jefe de Residentes, Servicio de Dermatología

${ }^{4}$ Médico de Planta, Servicio de Anatomía Patológica

${ }^{5}$ Médico de Planta, Servicio de Dermatología

6 Jefa, Servicio de Dermatología

Hospital Universitario Austral, Facultad de Ciencias Biomédicas, Universidad Austral, Pilar, Provincia de Buenos Aires, Argentina
}

Contacto de la autora: Carla Barbini

E-mail: carlabarbini1@gmail.com

Fecha de trabajo recibido: 16/3/2021

Fecha de trabajo aceptado: 12/6/2021

Conflicto de interés: los autores declaran que no existe conflicto de interés.

\section{INTRODUCCIÓN}

Un tumor de colisión es aquel conformado por dos poblaciones celulares diferentes, microscópicamente adyacentes una a la otra, pero que mantienen bordes diferenciados. Estas asociaciones incluyen tumores benignos, malignos o una combinación de ellos $^{1,2}$.

El mecanismo etiopatogénico que genera los tumores de colisión no está totalmente dilucidado; sin embargo, la teoría más aceptada postula que las dos neoplasias surgirían de clones celulares diferentes y su asociación sería solo una coincidencia ${ }^{3}$. Otra teoría sostiene que, en un área de piel fotodañada, aumenta el riesgo de aparición de distintos tumores adyacentes entre sí; por último, se propone la teoría de la interacción, según la cual, a través de un efecto paracrino, un tumor puede producir cambios estromales que inducirían a la formación de la segunda neoplasia ${ }^{1,4}$.

Se presentan tres casos de pacientes con tumores de colisión diagnosticados en el Hospital Universitario Austral y se realiza una revisión de la bibliografía. 


\section{SERIE DE CASOS}

\section{Caso clínico 1}

Un paciente de 78 años, con antecedentes de seis carcinomas basocelulares ubicados en el tronco, la frente y la región mandibular, consultó en el servicio de Dermatología, en febrero de 2006, por una lesión tumoral en la punta nasal, eritematosa, con una erosión central de unos $7 \mathrm{~mm}$, de bordes irregulares, con pigmento amarronado, de 8 meses de evolución. Se tomó una biopsia cuyo estudio histopatológico informó la presencia de un carcinoma basocelular (CBC) lobulado. Luego de su resección quirúrgica con la técnica micrográfica de Mohs, el nuevo informe anatomopatológico fue compatible con un $\mathrm{CBC}$ lobulado asociado a un lentigo maligno melanoma, con un espesor de Breslow de $0,75 \mathrm{~mm}$, Clark III y sin ulceración. Se realizó la ampliación de márgenes a $1 \mathrm{~cm}$ y la reconstrucción de la pirámide nasal con un colgajo mediofrontal e injerto de cartílago auricular. El paciente no presentó recurrencia en los controles durante los 10 años posteriores al diagnóstico (Fotos 1,2 y 3 ).

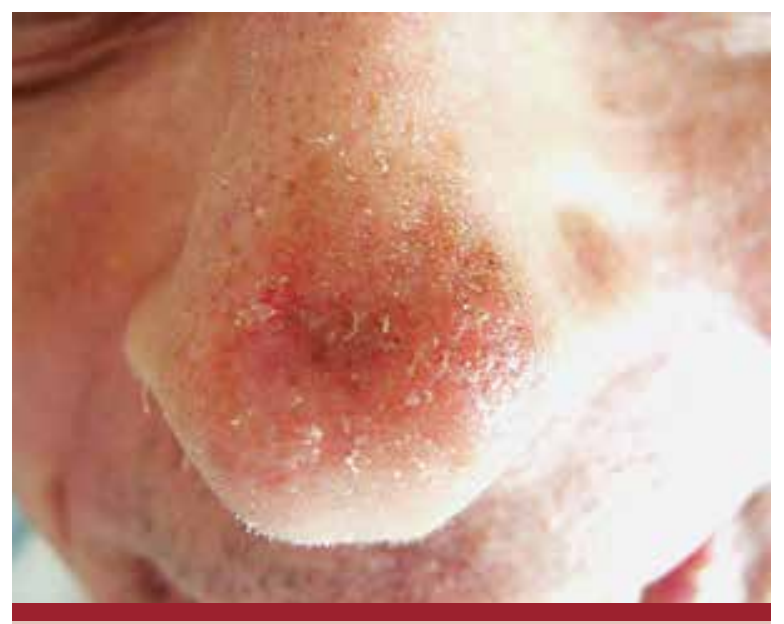

FOTO 1: Punta nasal, lesión tumoral maculosa, eritematosa, con erosión central de aproximadamente $7 \mathrm{~mm}$ de diámetro, de bordes irregulares con pigmento amarronado.

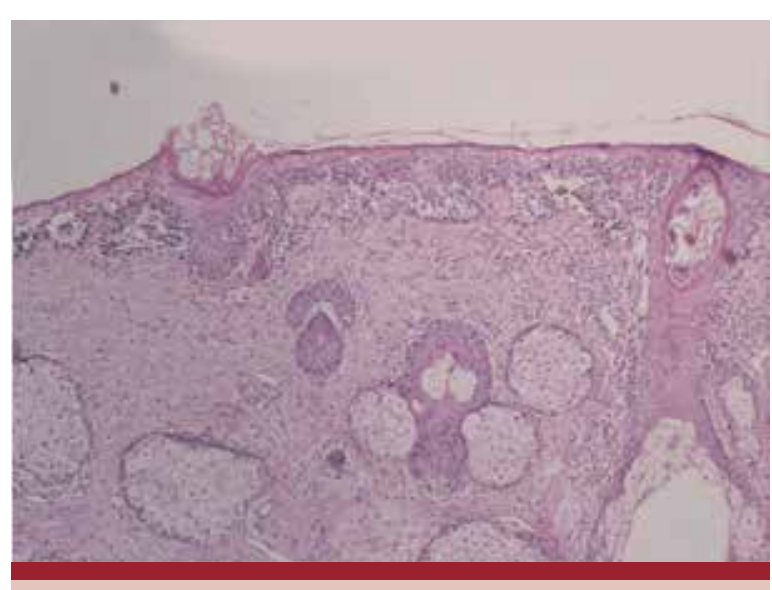

FOTO 2: Sector de corte histológico con lentigo maligno (HyE, 4X).

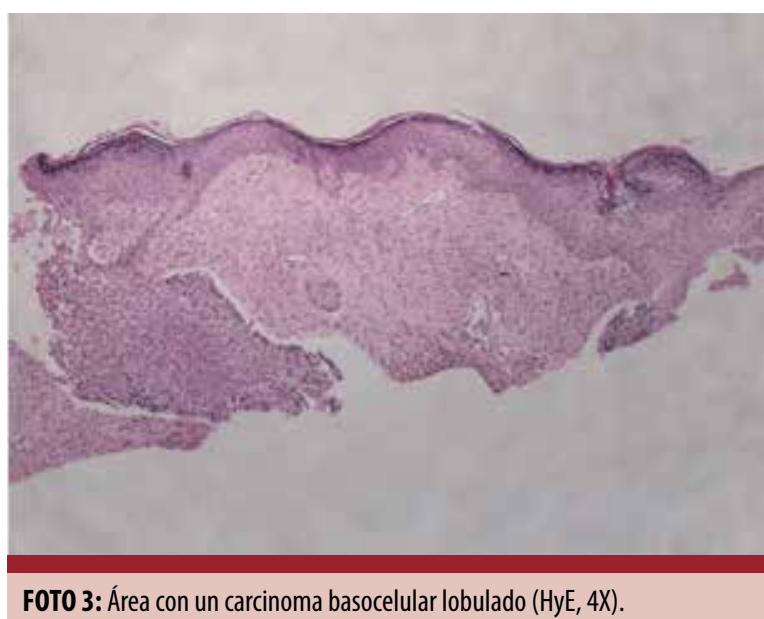

\section{Caso clínico 2}

Un paciente de 79 años, con antecedentes de dos carcinomas basocelulares en el dorso y en el hombro derecho consultó, en mayo de 2002, por la presencia de un tumor en la mejilla izquierda, en contacto con el párpado inferior, de 18 meses de evolución. Clínicamente, se observó una lesión tumoral de aspecto maculoso, pigmentada, de coloración marrón y negra, de $3 \mathrm{~cm}$ de diámetro, con áreas sin estructura y bordes irregulares. El estudio histopatológico informó la presencia de una neoproliferación melanocítica atípica y lentiginosa, que focalmente infiltraba la dermis papilar, compatible con un melanoma lentigo maligno, con un espesor de Breslow de 0,65 mm, Clark II, sin ulceración, asociado a un CBC metatípico. Se realizó la resección quirúrgica con un margen de $1 \mathrm{~cm}$ y la colocación de un injerto de piel de la región inguinal izquierda, con buena evolución y sin recurrencias (Fotos 4 y 5 ).

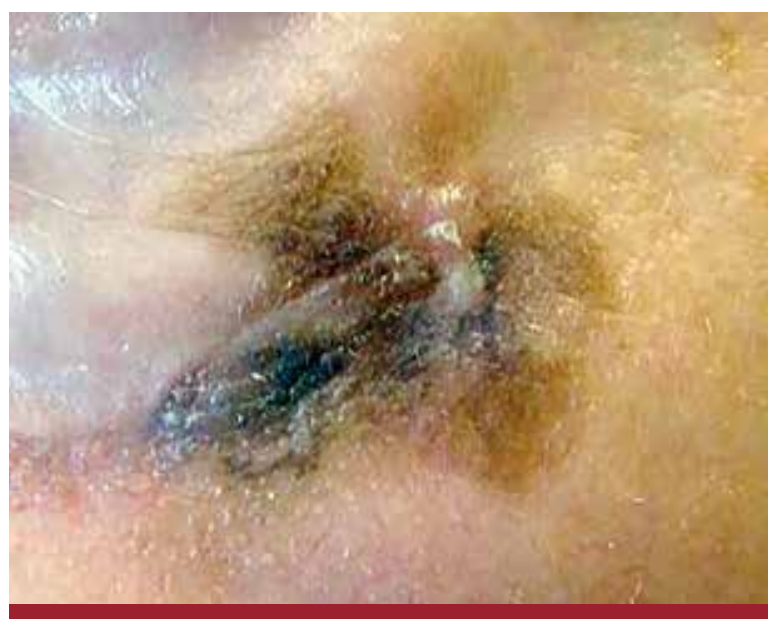

FOTO 4: Lesión tumoral pigmentada maculosa, con una red irregular marrón clara y áreas negras, sin estructura de distribución asimétrica y bordes irregulares, localizada en la mejilla izquierda. 


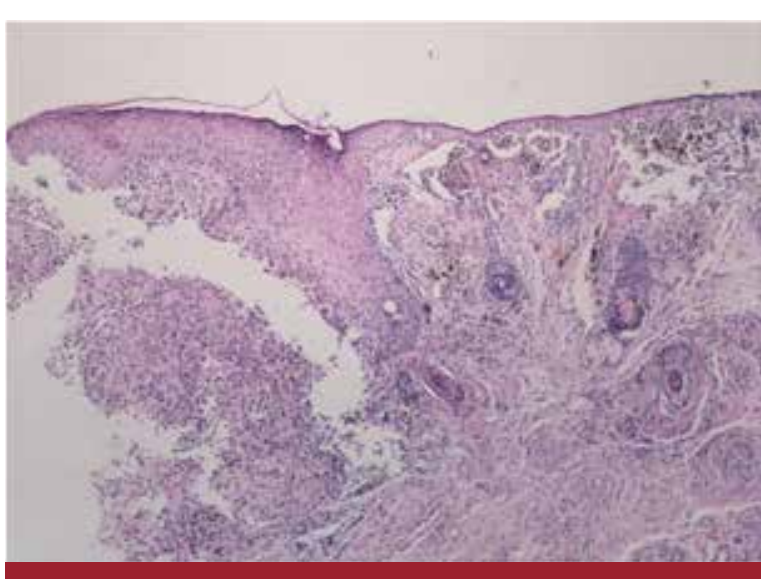

F0T0 5: Corte histológico que muestra a la izquierda un sector con un carcinoma basocelular metatípico y, a la derecha, un melanoma lentigo maligno (HyE, 10X).

\section{Caso clínico 3}

Una paciente de 54 años, con antecedentes de exposición solar intensa, consultó en el hospital, en julio de 2020, por la presencia de una lesión marrón-negruzca, localizada en el flanco derecho, de reciente aparición. En el examen físico se halló un tumor de aspecto maculoso, pigmentado, de $4 \mathrm{~mm}$ de diámetro, de bordes irregulares. En la dermatoscopia, se observaron dos sectores diferenciados: en el extremo medial, una red de pigmento regular y, en el extremo lateral, glóbulos negro-azulados de distintos tamańos con algunas áreas radiadas.

Se realizó la resección quirúrgica, cuyo estudio histopatológico confirmó la presencia de un tumor de colisión conformado por un CBC superficial pigmentado, asociado a un nevo de juntura lentiginoso (Fotos 6, 7, 8 y 9).

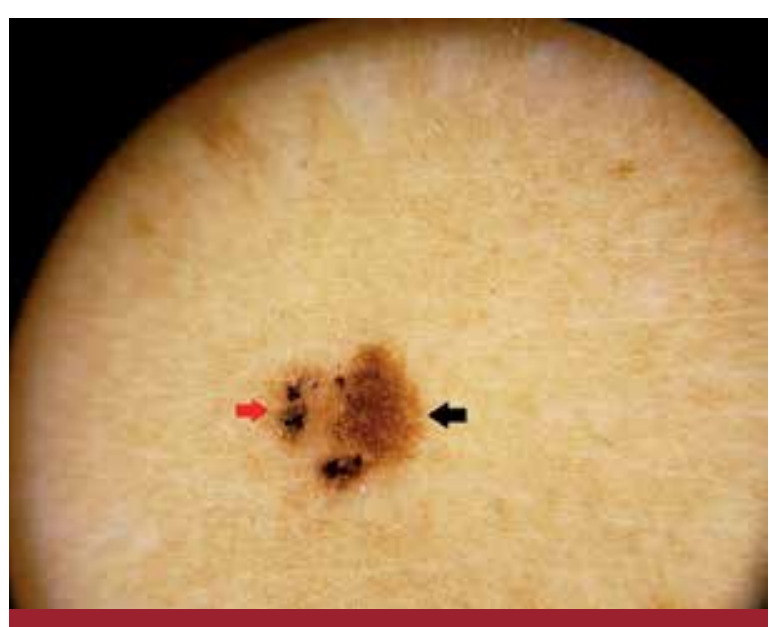

FOTO 6: Tumor pigmentado de $4 \mathrm{~mm}$ de diámetro y bordes irregulares en el abdomen. Flecha negra: red de pigmento marrón regular. Flecha roja: glóbulos negro-azulados de distintos tamaños y áreas radiadas.

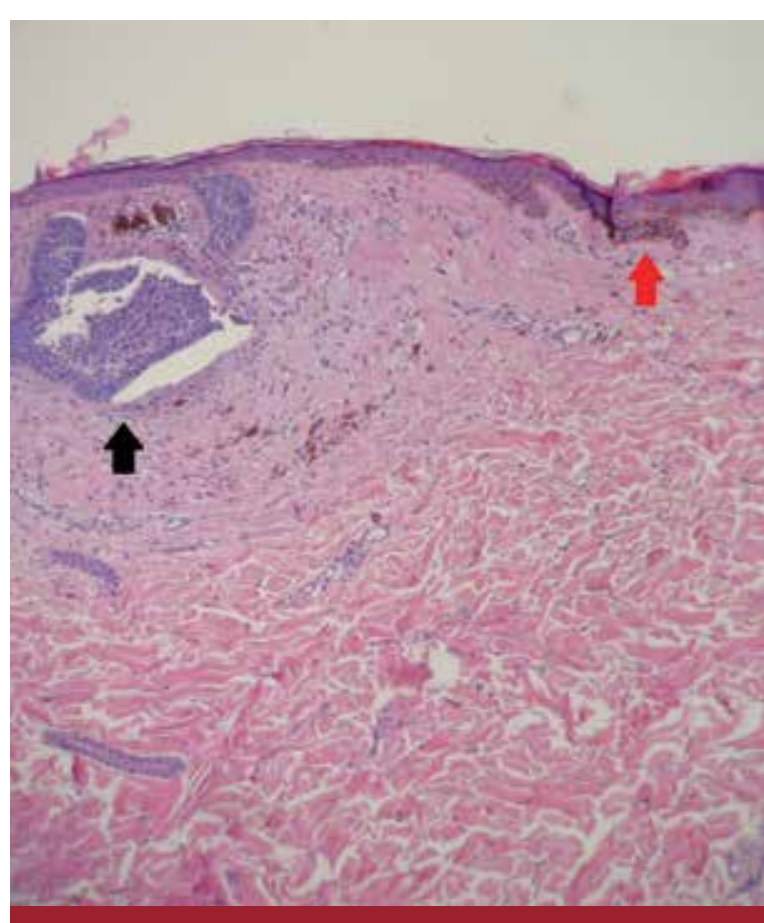

F0T0 7: Flecha negra: carcinoma basocelular superficial con un área de retracción y con la empalizada periférica característica. Flecha roja: nevo de juntura (HyE, 10X).

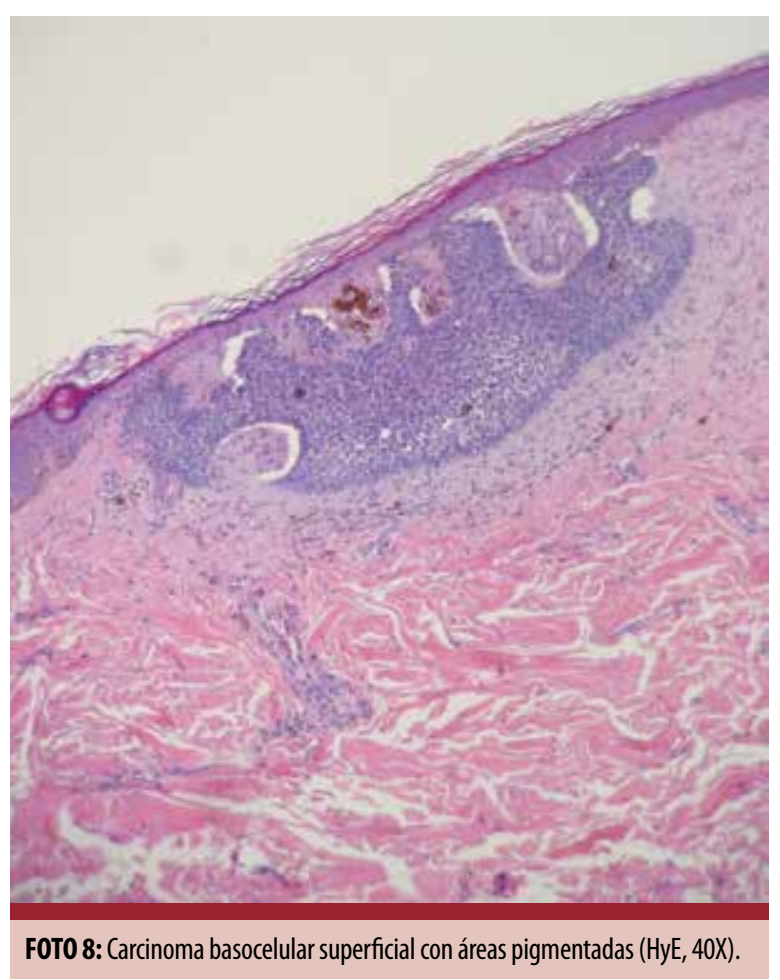

\section{DISCUSIÓN}

La primera publicación de un tumor de colisión que combinaba una neoplasia maligna melanocítica con otra maligna queratinocítica correspondió a Novick et ál. en 1977, cuando describieron un carcinoma esca- 


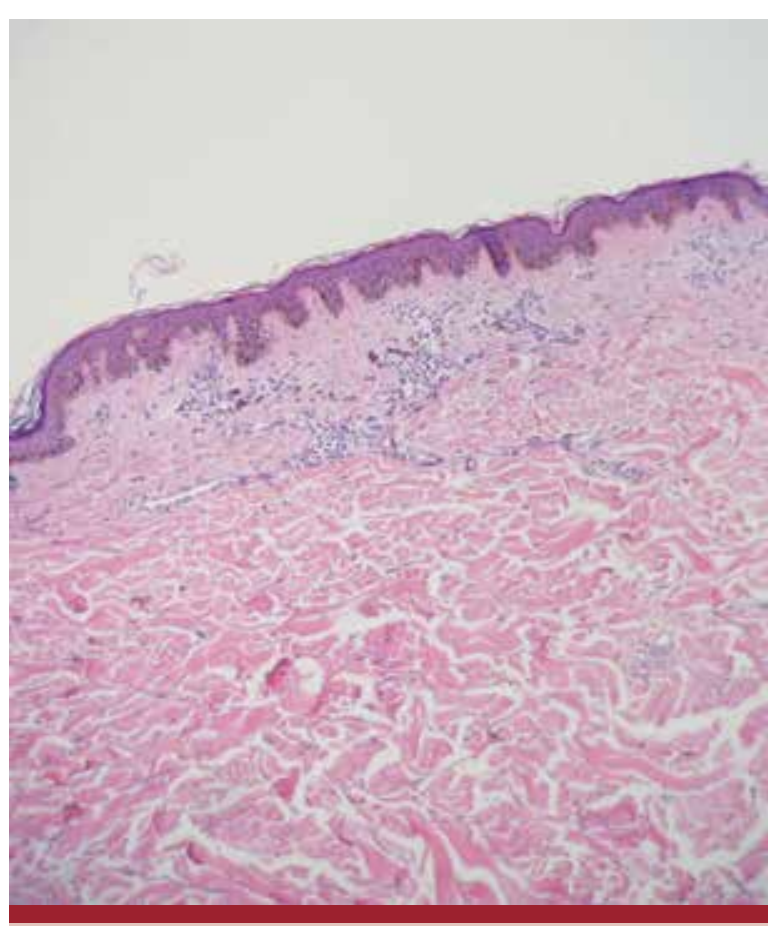

FOTO 9: Nevo de juntura con patrón lentiginoso y cambios involutivos (HyE, 10X).

moso asociado a un melanoma, sobre una cicatriz de quemadura previa 5 . En 1984, Bhawan et ál. publicaron el primer caso de colisión de un melanoma y un CBC, como ocurrió en dos de los pacientes analizados ${ }^{6}$.

En los pacientes menores de 60 años, es más común encontrar tumores de colisión con un componente melanocítico. En cambio, en los mayores de esa edad, es más frecuente el hallazgo de tumores de colisión con $\mathrm{CBC}$ o queratosis seborreicas. Esto se debería a que hay una reducción de los nevos con la edad, pero un aumento en la incidencia de tumores de distintas células epiteliales como el $\mathrm{CBC}$ o las queratosis seborreicas, entre otros ${ }^{7}$.

Esta rara ocurrencia de dos lesiones malignas en una misma muestra no debería llamar la atención, sobre todo en el caso de un CBC y un melanoma, dado que ambos se pueden desarrollar en una piel sometida a un daño solar crónico. Sin embargo, es un hallazgo poco frecuente ${ }^{8}$.

En un análisis de Boyd y Rapini, en el que se estudiaron 40.000 biopsias, se hallaron solo 69 casos de estos tumores de colisión. Las combinaciones más comunes incluyeron $\mathrm{CBC}$ con nevos (14 casos), nevo con queratosis seborreica (14 casos), $\mathrm{CBC}$ con queratosis seborreica (8 casos), queratosis actínica con nevo (7 casos), CBC con neurofibroma (4 casos), CEC con nevo (2 casos), CEC con dermatofibroma (2 casos), queratosis seborreica con quiste epidermoide (2 casos), cilindro- ma con espiradenoma ( 2 casos) y los 14 casos restantes fueron infrecuentes, con el hallazgo de una sola variante de distintos tipos de tumores de colisión. No se observaron asociaciones entre un $\mathrm{CBC}$ y un melanoma, como las vistas en los dos primeros casos comunicados ${ }^{9,10}$.

A diferencia de los resultados de ese estudio, se ha publicado en general que la asociación de dos tumores malignos más informada es la de un CBC y un melanoma ${ }^{1,3,11}$.

A pesar de la proximidad que presentan los queratinocitos y los melanocitos en la capa basal, los informes de tumores de colisión de estos orígenes han sido escasos ${ }^{1,3,9,11}$.

Habitualmente, la coexistencia de un melanoma y un $\mathrm{CBC}$ se produce en la cabeza, el dorso y los miembros y pueden presentarse en cualquiera de sus formas clínicas conocidas. Es frecuente que la lesión en colisión de estas dos neoplasias se asemeje clínicamente al CBC pigmentado ${ }^{12}$.

Los casos analizados de melanomas asociados a CBC se presentaron en la cara (punta nasal y mejilla) como lesiones tumorales pigmentadas, con la sospecha diagnóstica inicial de CBC en el caso 1 y de melanoma lentigo maligno en el caso 2. Fueron los resultados de los estudios anatomopatológicos los que describieron que ambas lesiones eran tumores de colisión.

Estos tumores deben diferenciarse de los tumores cutáneos combinados, que se originan de un mismo linaje celular y exhiben una diferenciación bidireccional o multidireccional ${ }^{13}$. Los casos más frecuentes son los tumores con varios tipos de diferenciación anexial o neuroendocrina. En estos tumores, es rara la combinación compuesta por melanocitos y queratinocitos. Se han publicado muy pocos casos de carcinomas escamosos combinados con melanoma (tumor escamomelanocítico) y de CBC con melanoma, también conocido como tumor basomelanocítico maligno , $^{313-15}$.

Además de la sospecha clínica, la dermatoscopia puede ser fundamental para aumentar la sensibilidad y la especificidad del diagnóstico, además que permite dilucidar la presencia de ambas lesiones aledañas según sus patrones dermatoscópicos específicos. En la paciente del tercer caso, se pudo observar la red de pigmento regular que se correspondió luego con la histopatología de un nevo de juntura y, en otro sector de la misma lesión, algunos de los criterios de $\mathrm{CBC}$ como la pigmentación marrón oscura observada como múltiples glóbulos y áreas radiadas en la periferia, en coincidencia con la descripción anatomopatológica del CBC superficial pigmentado.

Cabe destacar que la asociación que presentó la paciente de un nevo melanocítico y un carcinoma basocelular es la más frecuente dentro de los tumores de colisión compuestos por una neoplasia maligna y una benigna; 
incluso, el tumor maligno asociado con mayor asiduidad a lesiones melanocíticas benignas es el $\mathrm{CBC}^{1,16,17}$.

Cuando la dermatoscopia no define una única lesión y muestra patrones dermatoscópicos de dos o más entidades diferentes, es posible que se trate de un tumor de colisión. Se debe prestar especial atención si en una combinación benigna/maligna, la primera es más evidente y clara, ya que la sospecha diagnóstica puede estar equivocada ${ }^{1,18,19}$.

Otro instrumento que podría ser de utilidad junto con la dermatoscopia para el diagnóstico de los tumores de colisión es la microscopia confocal de reflectancia, ya que permitiría esclarecer las características de cada tumor y guiar de manera más detallada la toma de biopsia para efectuar el diagnóstico correcto ${ }^{20}$.

\section{CONCLUSIONES}

Los tumores de colisión son hallazgos infrecuentes que plantean desafíos diagnósticos y terapéuticos innegables.

\section{BIBLIOGRAFÍA}

1. Bulte CA, Hoegler KM, Khachemoune A. Collision tumors: $A$ review of their types, pathogenesis, and diagnostic challenges. Dermatol Ther. 2020;33e14236.

2. Gulia A, Altamura D, De Trane S, Micantonio T, et ál. Pigmented reticular structures in basal cell carcinoma and collision tumours. Br J Dermatol. 2010;162:442-444.

3. Cornejo KM, Deng AC. Malignant melanoma within squamous cell carcinoma and basal cell carcinoma: is it a combined or collision tumor: a case report and review of the literature. Am J Dermatopathol. 2013;35:226-234.

4. Rodríguez Saá S, Ciancio RM, Ruiz LR, Villa RE. Tumores en colisión: queratosis seborreica con nevus melanocítico. Estudio dermatoscópico e histopatológico a propósito de dos lesiones. Dermatol Argent 2011;17:405-408.

5. Novick M, Gard DA, Hardy SB, Spira M. Burn scar carcinoma: a review and analysis of 46 cases. J Trauma 1977; 17:809-817.

6. Bhawan J, Mehregan AH, Jung-Legg Y, Gellis SE. Pigmented basal cell carcinoma and superficial spreading malignant melanoma: an unusual combination. J Cutan Pathol. 1984;11:471-475.

7. Blum A, Siggs G, Marghoob AA, Kreusch J, et ál. Collision skin lesions-results of a multicenter study of the International Dermoscopy Society (IDS). Dermatol Pract Concept. 2017;7:51-62.

8. Papa G, Grandi G, Pascone M. Collision tumor of malignant skin cancers: a case of melanoma in basal cell carcinoma. Pathol Res Pract 2006;202:691-694.

9. Burkhalter A, White WL. Malignant melanoma in situ colonizing basal cell carcinoma. A simulator of invasive melanoma. Am J Dermatopathol. 1997;19:303-307.

10. Boyd AS, Rapini RP. Cutaneous collision tumors. An analysis of 69 cases and review of the literature. Am J Dermatopathol. 1994;6:253-257.
Son tumores que tienen presentaciones clínicas inespecíficas, lo que despierta dudas en el momento de la sospecha clínica diagnóstica. Esto puede llevar a sobreestimar la severidad de una lesión o a resecar innecesariamente un tumor de colisión compuesto por dos lesiones benignas.

Un recurso terapéutico actual es la dermatoscopia, que permite orientarse a lesiones benignas o malignas mediante la evaluación de las características de una lesión. En casos peculiares de algunos tumores de colisión, los signos dermatoscópicos pueden crear confusión por la combinación de patrones; esto debe alertar para la realización de la biopsia en el caso de sospechar lesiones malignas.

Presentamos los casos de 3 pacientes con tumores de colisión para recordar una entidad que, si bien es infrecuente, puede ser de difícil diagnóstico, lo cual podría conducir a un tratamiento insuficiente o inadecuado.

11. Piérard GE, Fazaa B, Henry F, Kamoun MR, et ál. Collision of primary malignant neoplasms on the skin: the connection between malignant melanoma and basal cell carcinoma. Dermatology. 1997;194:378-379.

12. Wang H, Benda PM, Piepkorn MW. Parasitism of basal cell carcinoma by lentigo maligna melanoma. J Am Acad Dermatol. 2003;48:S92-S94.

13. Rodriguez J, Nonaka D, Kuhn E, Reichel M, et ál. Combined high-grade basal cell carcinoma and malignant melanoma of the skin ("malignant basomelanocytic tumor"): report of two cases and review of the literature. Am J Dermatopathol. 2005;27:314-318.

14. Rosen LB, Williams WD, Benson J, Rywlin AM. A malignant neoplasm with features of both squamous cell carcinoma and malignant melanoma. Am J Dermatopathol. 1984;6:213-219.

15. Erickson LA, Myers JL, Mihm MC, Markovic SN, et ál. Malignant basomelanocytic tumor manifesting as metastatic melanoma. Am J Surg Pathol 2004;28:1393-1396.

16. Zaballos P, Llambrich A, Puig S, Malvehy J. Dermoscopy is useful for the recognition of benign-malignant compound tumours. Br J Dermatol. 2005;153:653-656.

17. Gulia A, Altamura D, De Trane S, Micantonio T, et ál. Pigmented reticular structures in basal cell carcinoma and collision tumours. Br J Dermatol. 2010;162:442-444.

18. Ansai S, Ogita A, Matsuda $H$, Saeki H, et ál. Collision of basal cell carcinoma and melanocytic nevus with unique dermoscopic findings. J Dermatol. 2016;43:584-585.

19. Zaballos-Diego P. Collision tumors. Actas Dermosifiliogr. 2014;105:310-311.

20. Moscarella E, Rabinovitz H, Oliviero MC, Brown L, et ál. The role of reflectance confocal microscopy as an aid in the diagnosis of collision tumors. Dermatology. 2013;227:109-117. 